\section{Economía de los medicamentos genéricos en América Latina}

\section{Federico Tobar $^{1}$}

Forma de citar: Tobar F. Economía de los medicamentos genéricos en America Latina. Rev Panam Salud Publica. 2008; 23(1):59-67.

Palabras clave: formulación de políticas, medicamentos genéricos, similar, América Latina.

\footnotetext{
1 Centro de Estudios en Gestión y Economía de la Salud, Facultad de Ciencias Económicas, Universidad de Buenos Aires. La correspondencia debe dirigirse a Federico Tobar, Calle Rodríguez Peña No. 608 (C1020ADN), Ciudad de Buenos Aires, Argentina. Correo electrónico: federico@federicotobar.com.ar
}

Los medicamentos son bienes de consumo, sin embargo, desde el punto de vista económico poseen algunas particularidades que los hacen específicos. Como bienes de salud constituyen el recurso médico y terapéutico más frecuentemente utilizado. Esto significa que las limitaciones en su producción, circulación y consumo pueden tener un importante impacto negativo en la salud de la población y en la distribución de las reservas de salud dentro de la sociedad. La principal consecuencia de tales limitaciones o fallas es el desajuste que se produce entre los recursos ofertados y las necesidades de salud de la población, lo que genera deficiencias en el acceso a los medicamentos (1).

El mercado de medicamentos es uno de los más complejos, debido a la cantidad de actores involucrados y la diversidad de papeles que estos desempeñan en el proceso que va desde la producción hasta el consumo. Aunque por sus características es difícil establecer comparaciones, este mercado presenta algunas analogías notables con otros sectores. Por ejemplo, su alto ritmo de innovación es similar al del mercado informático; la diversidad de los productos y el tamaño y las complejidades de las empresas productoras lo asemejan a la industria alimenticia; el volumen de facturación es comparable al de la industria bélica; y por la cantidad de intermediaciones - y en especial por el número de actores que participan en su financiación- es muy similar al sector de servicios médicos.

En la literatura especializada se han identificado varios factores que limitan la competencia o que generan una competencia imperfecta en el mercado de medicamentos, entre ellos la elevada capacidad que tiene la oferta para crear o inducir su propia demanda, su relación de agencia imperfecta -ya que quien prescribe toma las decisiones sobre su consumo en nombre del paciente, aunque no cuenta con toda la información ni toma siempre las decisiones más adecuadas en función del costo-, la amplia protección por patentes y la gran lealtad a la marca, además de la concentración oligopólica que existe por segmentos de productos. Sin embargo, todas estas manifestaciones realmente tienen una misma causa: las asimetrías en la información, que permite establecer una diferenciación artificial de los productos, de manera que aunque haya muchos fabricantes se mantiene la tendencia a mantener monopolios y a transformar a los consumidores en clientes cautivos. 
En este punto, vale la pena analizar con mayor detenimiento tres consecuencias importantes de esta fuerte diferenciación por marcas, característica de la producción, la circulación y el consumo de los medicamentos.

- La alta dispersión de precios. No hay ningún otro bien de consumo en el mercado mundial con tanta diferencia de precios entre diversos productos que responden a las mismas especificaciones técnicas. En este caso no se trata de un problema local ni regional. Tanto en países en desarrollo como en países desarrollados, la disparidad en los precios de los medicamentos llega a duplicar la de los alimentos y otros bienes industriales, como los automóviles y las máquinas herramientas. Esto se debe a una compleja lógica de producción, comercialización (que abarca la prescripción y la dispensación) y utilización de estos productos que propicia su diferenciación.

- Inelasticidad de los precios. La demanda de medicamentos no varía proporcionalmente con las variaciones de precio. En una situación de libre mercado y en presencia de una industria farmacéutica altamente concentrada, esta inelasticidad deja al consumidor en una situación de gran vulnerabilidad. Cuando la oferta depende de pocas empresas (oligopolios), estas mantienen cierto control sobre el precio y pueden elevarlo, pero eso no genera una gran disminución de la demanda. Un caso extremo es el consumo de insulina por parte de la población diabética: independientemente de su precio, el paciente no puede dejar de comprarla, aun cuando su precio aumente considerablemente. En el caso de otros bienes, como las entradas para el cine, la demanda disminuye proporcionalmente en la medida en que se incrementa su precio.

- Regresividad de su financiación. Los pobres gastan en medicamentos relativamente más que los ricos, porque tienen mayores necesidades que los ricos y disponen de menos condiciones para conseguirlos. Esta situación es mucho más grave en los países en desarrollo, donde una porción mayor de la financiación de los medicamentos depende de los ingresos de los hogares, es decir, el gasto en medicamentos en los países desarrollados se financia predominantemente con recursos públicos, mientras que en los países en desarrollo se financia mayormente con recursos privados.

Para superar el efecto de estas particularidades en la producción, comercialización y utilización de los medicamentos se han comenzado a incorporar nuevos esquemas regulatorios dirigidos a mejorar el acceso de la población a los medicamentos.

\section{Políticas de genéricos como modalidad de regulación}

Regular implica asumir que la armonía entre la oferta y la demanda no se logra por la operación de una "mano invisible" que ajusta los precios y permite una asignación óptima de los bienes y recursos y, por tanto, se necesitan normas e incentivos para corregir su funcionamiento. Pero regular el mercado de medicamentos no implica solamente resolver el problema del acceso de la población a los medicamentos esenciales, se necesita además garantizar la seguridad, la calidad y la inocuidad de esos productos. Aunque el tema del acceso siempre ha constituido un elemento importante en América Latina y ha generado diversas respuestas por parte de los gobiernos, las formas en que las regulaciones se conciben e implementan varían desde la limitación de la competencia hasta el respaldo total a la competitividad.

Hasta fines de la década de 1980, los Estados tendían a intervenir abiertamente, ya sea mediante el establecimiento de los precios, el otorgamiento de incentivos, la protección de la oferta de determinados productos o el aseguramiento del suministro con financiamiento público. Más tarde, durante la década de 1990 y como parte del denominado consenso de Washington, la mayoría de las economías de América Latina sufrieron un proceso de desregulación, del cual no escapó el mercado de medicamentos.

Se esperaba que sin la intervención estatal se estimulara la competencia y en consecuencia bajaran los precios. Sin embargo, eso no fue lo que sucedió. Durante las dos últimas décadas del siglo XX, el uso de medicamentos y el gasto mundial en las farmacias se incrementó en $7 \%$ anual, mientras en América Latina estos indicadores se mantuvieron estables e incluso retrocedieron levemente. Es decir, los precios aumentaron y el consumo disminuyó.

En contrapartida, los países desarrollados no realizaron una desregulación tan radical en materia de medicamentos y sus resultados en términos de acceso mejoraron. Entre las medidas regulatorias que aplicaron los países desarrollados se debe destacar la implementación de políticas sobre los medicamentos genéricos, ya que resultaron las herramientas más poderosas para lograr mejorar el acceso a los medicamentos y porque en lugar de restringir la competencia del mercado farmacéutico la fortalecieron.

\section{¿Qué es política de genéricos?}

Se utiliza la denominación de políticas de medicamentos genéricos para hacer referencia a un muy amplio conjunto de acciones. Pero todas ellas tienen en común el estar orientadas a crear un 
marco de competencia por precios en el mercado de medicamentos.

Resulta difícil definir qué se entiende por medicamento genérico debido a que los países utilizan diferentes definiciones para los mismos. En el caso particular de América Latina, Homedes y Ugalde registraron las grandes diferencias entre los criterios y exigencias que se aplican en diez países (2). Luego Vacca, Fitzgerald y Bermúdez compararon las políticas sobre medicamentos de 14 países latinoamericanos y confirmaron que se utilizan definiciones muy diferentes (3). Sin embargo, en general este término involucra al menos una dimensión legal, una fármaco-clínica y una económica.

Su dimensión legal está dada por el hecho de que se trata de medicamentos para los que no existen o han caducado los derechos exclusivos de comercialización (patentes). Esta es la base de algunas propuestas de denominarlos "productos competidores", aunque como Vacca y colaboradores advierten, en América Latina los mercados de productos competidores se consolidaron antes de la adopción de los acuerdos de propiedad intelectual, lo cual dificulta asumir esa denominación (3). La dimensión fármaco-clínica se manifiesta en que el medicamento genérico debe ser terapéuticamente equivalente al fármaco cuya seguridad y eficacia ha sido probada (llámese este medicamento de marca, original, bajo patente o de referencia). Una vez satisfechas las dimensiones legal y fármaco-clínica, se puede comercializar el producto genérico y establecer una competencia por precios, ya que al finalizar el período de protección que brinda la patente se eliminan los precios monopólicos. Es por este motivo que se puede hablar también de una dimensión económica de los medicamentos genéricos.

En la medida en que ingresen nuevos oferentes al mercado, la competencia por precios se fortalecerá y tenderán a converger en el llamado valor del producto (commodity value). Esto significa que los precios de estos productos se homogeneizarían a nivel internacional con valores muy próximos a su costo de producción y distribución en el mercado y, de esta forma, se minimizarían los márgenes de lucro, como ocurre con la materia prima. Esta dinámica competitiva en la que los mercados se abren a los productos genéricos conduce a la reducción de sus precios (4).

Si bien desde el punto de vista económico, el concepto de genérico implica que el medicamento compite con otros por precio y que la marca o nombre de fantasía pierde importancia, primero se debe demostrar la eficacia y la calidad homogénea de todos los productos similares presentes en el mercado, sean genéricos o no. Para ello es necesario contar con un buen sistema de farmacovigilancia y un riguroso esquema de registro. Sin embargo, aun- que se cumplan estas condiciones, queda un largo camino hacia la consolidación de un mercado competitivo que logre promover el acceso y aumentar al máximo los beneficios sociales. Desde el punto de vista económico, es posible clasificar las acciones y los pasos que se deben dar de la siguiente forma: 1) reducir las asimetrías en la información entre los productores, los prescriptores y los consumidores; 2) eliminar las barreras al ingreso al mercado; 3) establecer incentivos a la oferta; 4) establecer incentivos a la prescripción; 5) establecer incentivos a la demanda; y 6) establecer incentivos a la dispensación. A continuación se examinan las alternativas y desafíos para cada una de estas herramientas.

\section{Reducción de las asimetrías en la información}

Al analizar el "fetichismo de la mercancía", Karl Marx denunció el carácter hipócrita de la suposición de que todo comprador puede disponer de un conocimiento enciclopédico de los productos que adquiere. Los economistas neoclásicos reconocieron luego que la disponibilidad de información en tiempo y forma constituye una de las fallas más frecuentes del mercado (5). ${ }^{2}$ En el caso de los medicamentos, el problema es más complejo que en otros bienes, ya que quienes eligen el producto que se consume (los que los prescriben) no lo financian y quienes lo consumen (los pacientes) y financian (pacientes y aseguradores) no lo eligen. Los oferentes (laboratorios productores) suelen ser los únicos que poseen la información más completa en tiempo y forma, en tanto que el enfermo, como consumidor, se ubica en el extremo opuesto. A su vez, los prescriptores poseen en la práctica una información limitada que rara vez contiene nociones de precios y son el blanco de poderosas campañas publicitarias organizadas por los oferentes.

Para reducir las asimetrías en la información, el primer paso es exigir que en todas las etiquetas, prospectos y material publicitario de medicamentos aparezca la denominación común internacional del medicamento, también llamada denominación genérica. Esto no significa que se deba eliminar el

\footnotetext{
A fines del siglo XIX, algunos autores como Alfred Marshall, en Inglaterra, y León Walras, de la Escuela de Lausana, Suiza, entre otros, propusieron un modelo de equilibrio general de los mercados que constituye el núcleo del paradigma neoclásico. Aunque son múltiples los autores que les siguieron, vale la pena mencionar el giro importante que marcó Kenneth Arrow. En primer lugar, porque en 1954, a partir de su artículo Existence of an Equilibrium for a Competitive Economy, que escribió con G. Debreu (5), la teoría neoclásica centró sus esfuerzos en "acercarse a la realidad" señalando imperfecciones, fallas del mercado, asimetrías en la información, los bienes públicos, los efectos externos (externalidades), la incompletitud de los mercados, la competencia imperfecta, etc. En segundo lugar, porque en un trabajo publicado en 1963, Uncertainty and the Welfare Economics of Medical Care (5), introduce este análisis en el mercado de la nomics of Medical Care (5), introduce este análisis en el mercad
salud. Tales aportes le valieron el Premio Nobel en 1972.
} 
nombre comercial, sino que deben aparecer ambos: tanto el nombre de fantasía como el nombre real del medicamento. Al analizar los mecanismos reguladores en la Región de las Américas, Vacca y colaboradores constataron que este fue el aspecto más frecuentemente observado y estaba reflejado en las regulaciones vigentes en 13 de los 14 países estudiados (3). Se debe aclarar que esta es una medida que, al abarcar todos los medicamentos, favorece la competencia entre los medicamentos originales (o de referencia) y los productos genéricos. Sin embargo, mientras en algunos casos (como en México y Brasil) los denominados medicamentos genéricos o "genéricos de referencia" constituyen un mercado diferente de productos que son claramente distinguidos del resto, en otros (como por ejemplo, Argentina, Colombia y Chile) se engloba dentro de esta categoría a todos los productos similares (o copias).

El segundo paso es implementar mecanismos más rigurosos de regulación de la publicidad. La intensificación del control publicitario abarca medidas que van desde la prohibición de hacer propaganda directa al público en general de los productos de venta bajo receta, hasta la limitación de las muestras gratuitas.

Estas medidas no solo reducen las asimetrías en la información, sino que limitan la demanda inducida, que generalmente no es adecuada ni racional y aumenta innecesariamente los gastos. En los Estados Unidos de América, los gastos en anuncios directos al consumidor de medicamentos de venta bajo receta representan $15,7 \%$ de la facturación del mercado. Sin embargo, si a esa cifra se agrega el costo de la propaganda médica que se realiza mediante la entrega de muestras directamente a los prescriptores, el porcentaje asciende a 32\%. En Brasil, un informe de la Comisión Parlamentaria de Investigación sobre Medicamentos estimó que $20 \%$ de la facturación de los laboratorios se destina a la publicidad (6). Muchos países han avanzado en este sentido mediante la regulación de la publicidad, ya sea estableciendo límites o gravámenes impositivos adicionales (7).

Las grandes campañas masivas constituyen otro de los riesgos relacionados con la publicidad médica que amenazan la consolidación de mercados competitivos. A veces estas campañas anteceden al lanzamiento de los productos y por su formato consiguen eludir las restricciones para publicitar medicamentos de venta bajo receta. Se ha argumentado que tales campañas inducen en la población la adhesión a la marca desde el inicio del ciclo de vida del producto, lo que genera barreras que dificultan el ingreso de competidores en el futuro, sean estos variantes de medicamentos ya presentes en el mercado (a los que se denomina "me too"), similares o genéricos de referencia (8).
El tercer paso para reducir las asimetrías en la información es la fiscalización, ya que de nada sirve contar con normas pertinentes si no se realiza el control riguroso de su cumplimiento. El país de América Latina que ha mostrado mayores avances al respecto es Brasil, donde no solo se reglamentó la forma de hacer propaganda (9), sino que también se financió el examen de una muestra de 800 piezas publicitarias (cerca de $40 \%$ de las 2000 emitidas). Entre los resultados obtenidos se detectó que $80 \%$ de la propaganda presentaba irregularidades con respecto a la legislación. El monto recaudado por multas equivalió al costo de la fiscalización. En contraste, en Argentina se observó un retroceso en 2004, ya que la Agencia Nacional de Medicamentos, Alimentos y Tecnología (ANMAT) dejó de fiscalizar la publicidad con el argumento de la falta de presupuesto para ello (10).

La forma más exhaustiva de regulación de la publicidad de medicamentos sería establecer el requisito de habilitación previa de cada pieza publicitaria por parte de la autoridad regulatoria (11).

\section{Reducción de las barreras al ingreso al mercado}

La posibilidad de obtener mejores precios para un producto es directamente proporcional a la cantidad de oferentes del mismo. Por lo tanto, para consolidar un marco competitivo para el comercio de medicamentos es necesario facilitar la entrada de oferentes de genéricos al mercado. Esto requiere de un conjunto de medidas propias de la desregulación económica que facilitan la importación y flexibilizan el registro. El impacto de estas medidas, aplicadas desde inicios de la década de 1990 en varios países latinoamericanos, ha sido limitado y no han conducido a una mayor competencia (12). Los incentivos económicos en general y los incentivos al registro en particular fueron la información menos disponible y menos precisa en los documentos regulatorios de 14 países de la Región analizados (3).

Para lograr avances en la eliminación de las barreras a la competencia se deben adaptar los sistemas nacionales de registro. El registro es la base para autorizar la venta al público y su objetivo central es garantizar la calidad de los medicamentos circulantes en el mercado. Sin embargo, algunos países han comenzado a incorporar algunos criterios económicos para habilitar un nuevo producto. Visto desde la perspectiva del control de los precios y los gastos, se trata de una primera instancia de selección y, por lo mismo, un elemento clave en términos de eficiencia. El proceso de aprobación de un medicamento tiene un costo que se cubre con los aranceles que deben pagar los oferentes, por lo que en algunos países se ha pensado en flexibilizar los 
requisitos y acortar los plazos de aprobación. Sin embargo, esto no parece constituir realmente una barrera al ingreso de nuevos medicamentos en América Latina, ya que los aranceles que los oferentes deben pagar por el registro de un producto son hasta cien veces inferiores a los exigidos en los países desarrollados (12).

Otra de las medidas que pueden contribuir a reducir las barreras al ingreso de nuevos medicamentos es facilitar las importaciones. Si a los fabricantes nacionales les falta motivación para ofrecer medicamentos genéricos a precios accesibles, se pueden buscar fabricantes extranjeros interesados en hacerlo. Por eso adquiere una especial relevancia la propuesta de homologación de los registros de medicamentos de países con niveles similares de control, así como la adopción de requisitos comunes para el registro de medicamentos. Esta medida se puede implementar mediante decretos presidenciales y el registro podría aprobarse de forma automática en un plazo mínimo ante la presentación por parte de los solicitantes del conjunto mínimo de requisitos propuestos por el Grupo Técnico de la Organización Mundial de la Salud para el Registro de Medicamentos (13). En Brasil, por ejemplo, el Decreto 3675/00 aprobó el registro provisional de genéricos importados que estuvieran registrados en los Estados Unidos, Canadá o algunos países europeos (14). En Argentina, desde 2002, se habilitó la importación directa de medicamentos genéricos mediante la homologación del registro del país de origen, aunque no para su comercialización general, sino exclusivamente para el Programa Remediar, dirigido a la provisión pública de medicamentos.

Una herramienta regulatoria de mayor impacto sobre el acceso consiste en controlar los precios en el momento del registro. Los países europeos y Brasil han avanzado en esa dirección y, por ejemplo, en este último país los medicamentos genéricos resultan $35 \%$ más baratos en el momento de su lanzamiento que el medicamento de referencia. Sin embargo, se debe tener presente que, en rigor, esta medida no reduce las barreras al ingreso de medicamentos, sino que aumenta el control directo sobre los precios, por lo que responde más al antiguo modelo intervencionista que a esquemas que promueven la competitividad.

La armonización de las normas en general y la incorporación de las buenas prácticas de manufactura en particular también pueden introducir barreras al ingreso de medicamentos genéricos al mercado. Con el pretexto de avanzar hacia controles de calidad de producción más rigurosos se puede terminar incorporando restricciones a la oferta de firmas pequeñas o de capital nacional. Por ese motivo, la incorporación de estas regulaciones debe ser gradual y programada. En América Latina ya se cuenta con una masa crítica de profesionales y un nivel adecuado de desarrollo que permitirán llevar a cabo esa incorporación progresiva.

La estrategia más radical para ampliar la oferta de productos genéricos y minimizar las barreras a su ingreso consiste en habilitar todos los productos registrados en el país para operar como genéricos. Esto es lo que se hizo en Argentina mediante la Ley 25.649 de 2002 (15), que obliga a utilizar todos los medicamentos según su denominación común internacional. En este caso, en lugar de distinguir los medicamentos genéricos de los similares se los equipara y en lugar de crear un nuevo mercado de medicamentos genéricos se logra que todos los productos compitan por su precio. El impacto sobre la contención de precios que se puede lograr por esta vía será directamente proporcional a la dispersión de precios vigente en el mercado. Por ejemplo, si en un país hay 10 marcas (alternativas comerciales) registradas de amoxicilina de $500 \mathrm{mg}$ en cajas que contienen blísteres de 30 comprimidos cuyos precios varían de US\$ 3,00 a US\$ 12,00 por caja, la utilización del medicamento por su denominación común internacional impide que la receta médica induzca a comprar la marca más cara (que detenta precios monopólicos) y el paciente puede decidir comprar la versión más económica del producto. Sin embargo, es probable que a medida que la demanda aumente para unos productos y disminuya para otros, los precios tiendan a converger, por lo que el ahorro logrado resultará decreciente.

\section{Incentivos a la oferta}

Se consideran incentivos a la oferta las medidas que el gobierno implementa para estimular la fabricación y la venta de medicamentos genéricos. Cuando en un mercado de genéricos los precios de competencia tienden a converger con el costo del producto, se generan condiciones ideales para promover el acceso al mercado. Sin embargo, puede faltar motivación a los fabricantes para mantener o incrementar su producción, por lo que es necesario incorporar nuevos incentivos a la oferta, aun si ya existieran oferentes de productos genéricos.

Entre las medidas más frecuentemente empleadas para elevar la motivación de los productores están las disposiciones de promoción industrial, como la de reducir -o incluso eliminar- los impuestos a los productos genéricos y los aranceles de importación de principios activos y diversos insumos, entre otros. Sin embargo, estas acciones no se han utilizado para promover el acceso de la población a los medicamentos.

Otra vía de motivación es la restricción de la vigencia de las patentes. En 1995, un acuerdo co- 
mercial adoptado en el marco los Aspectos de los Derechos de Propiedad Intelectual Relacionados con el Comercio (ADPIC) estableció, entre otras cosas, que los países en desarrollo que lo suscribían se comprometían a respetar las patentes de los productos por un plazo de veinte años (16). Más tarde, en la declaración final de la Ronda de Doha, Qatar, en 2001, la Organización Mundial del Comercio (OMC) aprobó permitir a algunos países miembros flexibilizar algunos aspectos relacionados con el reconocimiento de las patentes. En su párrafo $7 .^{\circ}$ se establece que los países menos desarrollados que aún no lo han hecho, tienen un plazo hasta 2016 para extender la protección de la propiedad intelectual a productos y procesos farmacéuticos (17). India ha mostrado una gran habilidad para utilizar todo el período de transición para fortalecer su capacidad tecnológica.

Una alternativa adicional consiste en hacer uso de la llamada "Cláusula Bolar", que permite iniciar los estudios requeridos para registrar un producto antes del vencimiento de su patente. Aunque esta podría considerarse una medida que derriba barreras al ingreso de genéricos al mercado, en realidad lo que hace es acelerar la habilitación de posibles oferentes para producir y comercializar versiones genéricas de un producto protegido aún por una patente. Los gobiernos de algunos países desarrollados, como los Estados Unidos, Japón, Australia y Canadá, han hecho de este recurso una herramienta política para fortalecer su oferta de genéricos.

No obstante, la medida contemplada en los ADPIC que más incentiva la oferta local de genéricos es la implementación de licencias obligatorias. Esto significa que, aun sin el consentimiento del titular de la patente, un gobierno puede autorizar la producción de un determinado medicamento por diversas razones, como la falta de producción local o una emergencia nacional. Canadá, por ejemplo, ha empleado este mecanismo para fortalecer la producción nacional de genéricos (12). Otro incentivo indirecto a la oferta de genéricos es incorporar barreras al ingreso de nuevos productos que no presenten ventajas económicas. Esto desestimula la llamada pseudoinnovación y las innovaciones que encarecen los tratamientos. Para implementar esta medida es necesario exigir la presentación de estudios farmacoeconómicos como parte del proceso de registro.

Una modalidad extrema para garantizar la disponibilidad de genéricos consiste en no recurrir a su provisión por el mercado, sino mediante la producción pública. Aunque no se trata de una medida que promueve la competencia, sino de una respuesta intervencionista a la vieja usanza, puede ser una poderosa vía para facilitar el acceso de la población a los medicamentos. Las políticas de producción pública de medicamentos genéricos en México, Chile y Brasil tuvieron un considerable efecto en este sentido, mientras en Brasil las denominadas "farmacias populares" comercializan genéricos de producción pública. Es por ello que, aun en la actualidad, a veces se asocia el concepto de medicamento genérico con la producción pública.

Es posible identificar cuatro combinaciones de producción y circulación de genéricos: a) la producción pública para su circulación exclusiva en los servicios públicos, por ejemplo, cuando los hospitales fabrican algunos de los productos que se utilizan dentro del mismo establecimiento sin requerir de registro ni habilitación comercial ; b) la producción pública con alternativas para su comercialización, por ejemplo en Brasil se venden al público a través de las Farmacias Populares productos fabricados en laboratorios oficiales (como Far Manguinhos); c) la producción privada para su circulación exclusiva en los servicios públicos, por ejemplo, el Programa Remediar de Argentina y el Programa de Cuidados Sanitarios Básicos de Paraguay compran mediante licitaciones internacionales medicamentos genéricos que son provistos en envases diferenciados en los que no figura ningún nombre comercial y se destaca en el rótulo que son para uso exclusivo en los servicios públicos de salud y se penaliza su venta o uso en los servicios privados; y d) el establecimiento de un mercado de genéricos mediante la producción y la comercialización privadas, que constituye la alternativa más frecuente.

\section{Incentivos a la prescripción}

Un elemento central para la incorporación de las políticas de medicamentos genéricos es garantizar su aceptación por parte de la sociedad, es decir, la consolidación de una oferta relativamente adecuada a las necesidades de salud de una población no tendrá un impacto positivo si las personas no demandan los productos. Por lo tanto, resulta fundamental aplicar medidas que promuevan la demanda de medicamentos genéricos, especialmente las dirigidas a los profesionales que prescriben los medicamentos, por ser quienes deciden, en principio, el consumo y la utilización de los medicamentos. Por ejemplo, los seguros de salud pueden otorgar premios económicos a los profesionales que prescriben genéricos con mayor frecuencia. En realidad se pueden utilizar los mismos incentivos que ha utilizado tradicionalmente la industria de medicamentos de marca para los profesionales encargados de la prescripción de productos farmacéuticos.

En ocasiones se han aplicado medidas alternativas a las desarrolladas por la industria, como la 
información y actualización de los profesionales. De hecho, se puede postular que la reducción de las asimetrías en la información que disponen los médicos constituye un incentivo a la prescripción de genéricos. En otros términos, se trata de facilitar y orientar la gestión clínica mediante la medicina basada en la evidencia. En ese sentido, los formularios terapéuticos contribuyen a promover la prescripción y la utilización de los medicamentos de la forma más objetiva posible.

La forma extrema de promover la prescripción según el nombre genérico es establecer su obligatoriedad por ley. Aunque esto está contemplado en la legislación de cinco países latinoamericanos (Argentina, Ecuador, Panamá, Paraguay y Perú) (3), en esos países no se vigila el cumplimiento de la ley a nivel de las farmacias ni se sanciona a quienes la incumplen. Además, los tratados de libre comercio suscritos por varios países de América Latina con los Estados Unidos contienen cláusulas que restringen el uso de la denominación común internacional.

\section{Incentivos a la demanda}

Además de los incentivos a la prescripción, también hace falta que el comprador se decida a adquirir medicamentos genéricos, ya se trate de compradores individuales o institucionales.

Una frecuente y poderosa medida es el establecimiento de precios de referencia. Esto consiste en fijar un precio máximo por producto para la cofinanciación o el reembolso por parte del gobierno o de los agentes de seguro de salud. En algunos países como España, el precio de referencia que reconoce la seguridad social es el de los medicamentos genéricos disponibles en el mercado. Si los pacientes optan por alternativas comerciales más caras deben costear la diferencia con sus propios recursos.

De manera similar a lo establecido en la mayoría de los países de Europa, las instituciones de seguridad social de Argentina establecieron precios de referencia en el año 2002. Además, mediante la resolución 163/2002 de la Superintendencia de Servicios de Salud de Argentina (18), se estableció que los agentes de seguro de salud reconocerán y aplicarán los descuentos a favor de los beneficiarios solamente cuando las prescripciones de medicamentos que emitan los servicios ambulatorios se hagan por el nombre genérico. El impacto de estas medidas en la reducción del gasto en medicamentos puede ser muy elevado. Por ejemplo, un adulto con hipertensión arterial e hipercolesterolemia que hubiera seguido un tratamiento con enalapril y simvastatina en Argentina durante 2004, habría tenido un gasto mensual de \$290,10 (pesos argentinos) si utilizaba las marcas de mayor costo mientras que el gasto habría sido solamente de $\$ 40,03$ si empleaba los dos productos genéricos más económicos. Esto significa un ahorro anual para el paciente de $\$ 3480$,00, equivalentes a US\$1 150,00, según el cambio oficial vigente en 2004.

En América Latina ya se ha generalizado la práctica de que las institucionales realicen sus compras exclusivamente mediante la denominación común internacional. Sin embargo, el arsenal de medidas para promover la competencia y consolidar el uso de medicamentos genéricos es más amplio, y son grandes las posibilidades de promover la competencia en la adecuada selección de los productos (que se basa en las especificaciones técnicas). Por ejemplo, cuando se trata de medicamentos para los que aún no se dispone de versiones genéricas, se puede hacer competir a dos o más productos de marca y se puede ampliar la competencia mediante la incorporación de los denominados productos "me too". Entre las medidas adicionales incorporadas en el Programa Remediar, en Argentina, se exige utilizar envases empadronados en los que no figure el nombre de fantasía ni las marcas originales de los productos.

\section{Incentivos a la dispensación}

La principal medida para incentivar la dispensación de los productos genéricos es permitir a los profesionales farmacéuticos substituir el medicamento de marca prescripto en la receta por uno genérico cuando el principio activo es idéntico y la presentación (en número de dosis) es equivalente. Esta medida se puede reforzar si se provee a los profesionales de los dispensarios la información de las equivalencias de los productos.

Sin embargo, esto no siempre es suficiente, ya que las ganancias de los farmacéuticos son proporcionales al precio del medicamento y esta habilitación podría inducirlos a hacer la substitución por equivalentes más caros y no más económicos. Por eso, se deben establecer, además, incentivos especiales que promuevan la dispensación de medicamentos genéricos. En Holanda, por ejemplo, se implementó una bonificación por dispensar medicamentos genéricos de menor precio que los de marca. Las instituciones sanitarias denominadas Health Maintenance Organizations, de los Estados Unidos, establecen primas o bonos por dispensar genéricos o alguno de los medicamentos incluidos en una lista de medicamentos cuya prescripción se busca incentivar, además de premios a las farmacias en las que más cantidad de medicamentos de esta clase se dispensen.

Finalmente, para reforzar los incentivos a la dispensación se pueden establecer modelos de re- 
muneración a los farmacéuticos que no se basen en un porcentaje fijo sobre el precio del producto. Por ejemplo, se podría pagar por acto farmacéutico (monto fijo por receta o producto dispensado) o un monto mensual por paciente.

\section{CONCLUSIONES}

La inflación de los precios de los productos farmacéuticos, asociada con la reducción del acceso de la población a los medicamentos, es resultado de las políticas desregulatorias adoptadas en muchos países. Estas políticas no han generado soluciones ni ventajas desde el punto de vista social y sanitario. La formación de los precios de los medicamentos no solo está relacionada con la eficiencia sino también, de manera muy especial, con la equidad. En otras palabras, si se modifican los precios de los medicamentos se modifica la forma en que la salud se distribuye en la sociedad.

Ante este problema, se han ensayado diferentes tipos de medidas que grosso modo se pueden resumir en dos modelos de intervención bien diferenciados. Por un lado, la adopción de estrategias intervencionistas que en general optan por limitar la competencia con medidas tales como el establecimiento de precios fijos. Por el otro, la aplicación de políticas que promueven la competencia y que fomentan una dinámica de mercado tal, que los resultados producen beneficios sociales.

Las políticas de genéricos se enmarcan en este segundo grupo y constituyen una modalidad de políticas de regulación del mercado de medicamentos en la que intervienen tanto medidas de promoción como incentivos a la producción y al uso de los medicamentos genéricos. Para ello se establecen mecanismos activos en las diferentes etapas del proceso de abastecimiento de medicamentos: producción, registro, dispensación, prescripción y utilización.

No existe un modo unívoco de promover el empleo de los medicamentos genéricos. Se puede favorecer su incorporación al mercado mediante un amplio conjunto de alternativas. Estas alternativas se han clasificado y analizado en dependencia de las fallas o limitaciones del mercado que se deben superar. Dentro de cada una de las dimensiones analizadas, se han identificado diferentes opciones, en dependencia de la magnitud de la consolidación del mercado de medicamentos genéricos que se quiere lograr.

\section{SYNOPSIS}

\section{Economy of generic drugs in Latin America}

In terms of economics, implementing generic drug policy is nothing other than consolidating (or even, creating) drug markets that set competitive prices, the result of which favors public access to essential drugs. This article approaches the topic of generic drugs from the economic perspective by examining various regulatory models in order to evaluate and leverage generic drug policy implementation options as a mechanism for battling some of the markets' specific weaknesses. The conclusion is that there is no single unequivocal method for promoting the use of generic drugs, and that the most favorable way to integrate markets may be through a broad combination of alternatives. These alternatives are grouped and analyzed according to the market issues or challenges that must be overcome. Several options are then identified based on the degree of market consolidation to be obtained.

Key words: policy making; drugs, generic; Latin America.

\section{REFERENCIAS}

1. Luiza VL, Bermúdez J. Acceso a medicamentos: conceitos e polémicas. En: Bermúdez J, Oliveira MA, Esher A. Acceso a medicamentos: derecho fundamental, papel del Estado. Rio de Janeiro: ENSP. 2004. Pp. 45-67.

2. Homedes N, Ugalde A. Multisource drug policies in Latin America: survey of 10 countries. Bul World Health Organ. 2005;83:64-70. Hallado en: http:/ /www. who.int/bulletin/volumes/83/1/64. pdf. Acceso el 27 de octubre de 2007.

3. Vacca González P, Fitzgerald J, Bermúdez J. Definición de medicamento genérico ¿un fin o un medio? Análisis de la regulación en 14 países de la Región de las Américas. Rev Panam Salud Publica. 2006;20(5):314-23. Hallado en: http:// www.scielosp.org/pdf/rpsp/v20n5/ 04.pdf. Acceso el 27 de octubre de 2007.

4. Creese A, Quick J. Differential pricing arrangements and feasibility: context setting paper. Geneva: World Health Organization; 2001

5. Arrow K. Collected Papers of Kenneth Arrow. General equilibrium. Vol 2. Cambridge: Belknap Press, Harvard University Press; 1983.

6. Nascimento A. Ao persistirem os síntomas o médico deverá ser consultado: isto é regulação? São Paulo: Sociedade Brasileira de Vigilância de Medicamentos; 2005.

7. Mossialos E. El impacto sobre los medicamentos de la contención del gasto y las reformas en la asistencia sanitaria. En: Lobo F, Velázquez G, eds. Los medicamentos ante las nuevas realidades económicas. Organización Mundial de la Salud, Universidad Carlos III de Madrid, Editorial Civitas, SA; 1997. Pp. 109-50. Hallado en: http://www.who. int $/$ medicinedocs $/$ library.fcgi? $=d$ 010edmweb-000-1-0-010-4-00-101-11en-5000-50-help-0-01131- 
001-110utfZz-8-0-0\&cl=CL1.1.7\&d= Jh2925s\&gt=2\#Jh2925s.6.1. Acceso el 27 de octubre de 2007.

8. Frenkel, J. O mercado farmacêutico brasileiro: a sua evolução recente, mercados e preços. En: Negri B, di Giovanni G, eds. Brasil: radiografia da saúde. Campinas, SP: UNICAMP, Instituto de Economia; 2001.

9. Directoría Colegiada de la Agencia Nacional de Vigilancia Sanitaria. Resolución $\mathrm{N}^{\circ}$ 102/2000. Brasilia: Agencia Nacional de Vigilancia Sanitaria; 2000.

10. República Argentina, Ministerio de Salud y Ambiente. Resolución No. 20/ 2005. Publicidad sobre productos de venta libre. Buenos Aires: Ministerio de Salud y Ambiente. Hallado en: http:// www.infoleg.gov.ar/infolegInternet/ anexos/100000-104999/103157/norma. $\mathrm{htm}$. Acceso el 27 de octubre de 2007.

11. Moura D. Saúde não se dá, conquista-se. São Paulo: Editora Hucitec; 1989

12. Tobar F. Estrategias económicas y financiamiento del medicamento. En: Bermúdez J, Oliveira MA, Esher A. Acceso a medicamentos: derecho fundamental, papel del Estado. Rio de Janeiro: ENSP; 2004. Pp. 103-37.

13. Organización Panamericana de la Salud, Grupo de Trabajo en Registro de Medicamentos. Informe y propuesta sobre requisitos comunes para el registro de medicamentos. IV Conferencia Panamericana para la Armonización de la Reglamentación Farmacéutica; Santo Domingo, República Dominicana; 2-4 de marzo de 2005. Washington, D.C.: OPS; 2005. (Doc IV-4 GT/Registro de Medicamentos). Hallado en: http://www. paho.org/Spanish/AD/THS/EV/ IVCONF_Registro-Med-esp.doc. Acceso el 17 de septiembre de 2007.

14. República de Brasil. Decreto 3675/00. Dispõe sobre medidas especiais relacionadas com o registro de medicamentos genéricos. Brasília: Ministério de Saúde; 2000. Hallado en: http://www.cff. org.br/Legisla $\%$ C3\%A7\% $\% 3 \% \mathrm{~A} 30 /$ Decretos/dec_3675_2000.html. Acceso el 27 de octubre de 2007.

15. República Argentina. Ley 25649. Especialidades medicinales. Buenos Aires: Ministerio de Salud y Ambiente; 2002.
Hallado en: http://www.infoleg.gov. $\mathrm{ar} /$ infolegInternet/anexos /7500079999/77881/norma.htm. Acceso el 27 de octubre de 2007.

16. Bermúdez J, Oliveira M, Chaves G. O acordo TRIPS da OMC e os desafios para a saúde Pública. En: Bermúdez J, Oliveira MA, Esher A. Acceso a medicamentos: derecho fundamental, papel del Estado. Rio de Janeiro: Escola Nacional de Saúde Pública Sergio Arouca; 2004. Pp. 75.

17. Programa de Trabajo de Doha. Declaración Ministerial. 22/12/2005. Hallado en: http://www.wto.org/spanish/ thewto $s /$ minist_s/min 05 s/final text_s.htm. Acceso el 27 de octubre de 2007.

18. República Argentina, Ministerio de Salud y Ambiente. Resolución 163/2002. Medicamentos en modalidad ambulatoria. Buenos Aires: Ministerio de Salud y Ambiente; 2002. Hallado en: http:// www.infoleg.gov.ar/infolegInternet/ anexos/70000-74999/74213/norma. $\mathrm{htm}$. Acceso el 27 de octubre de 2007. 